\title{
The long-chain alkenones in the upper layers of bottom sediments of salt lakes in Southern Siberia as a potential biomarker of paleo-climate
}

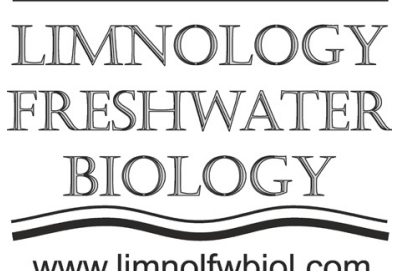

www.limnolfwbiol.com

\author{
Bulkhin A.O. ${ }^{1,2}$, Rogozin D.Yu. ${ }^{1,2 .}$ \\ ${ }^{1}$ Institute of Biophysics, Siberian Branch of the Russian Academy of Sciences (IBP SB RAS), Akademgorodok Str., 50/50, Krasnoyarsk \\ region, Krasnoyarsk, 660036, Russia \\ ${ }^{2}$ Siberian Federal University (SibFU), 79 Svobodny Ave, Krasnoyarsk region, Krasnoyarsk, 660041, Russia
}

\begin{abstract}
The long-chain alkenones in the upper layers of the bottom sediments of ten salt lakes in Southern Siberia were collected as a bio-marker for paleo-limnological studies in the summer 2019. The quantitative and qualitative composition of long-chain alkenones were determined by means of gas chromatography mass spectrometry. Also, the possibility of using this class of compounds as a saltsensitive bio-marker for paleoclimatic reconstructions in the corresponding regions was assess. The results showed that the extracted alkenones are a mixture of several fractions with different lengths of the carbon chain and the number of double bonds. The alkenone C37:4 fractions positively correlate with the salinity of the studied lakes and can serve as a marker of salinity in paleo-climatic reconstructions.
\end{abstract}

Keywords: Alkenones, paleoproxies, paleoclimatology, haptophyta algae, bottom sediments.

\section{Introduction}

Biochemical markers of paleo-climate is a promising area of research for paleo-limnological reconstruction. Alkenones may serve as a biochemical indicator of thermal and salinity paleo-conditions. Alkenones, is a class of C37-C40 polyunsaturated long chain hydrocarbons with 2, 3 and 4 trans double bonds located at intervals of 7 carbon atoms and a keto group on the 2nd (ME ketone) or 3rd (ET ketone) carbon of the aliphatic chain (Araie et al., 2018). These compounds are synthesized only by haptophytic microalgae of the order Isochrysidales. Those compounds are ubiquitously found in the marine environment, particularly sediments, and the alkenone unsaturation indices (UK37 and UK'37) have been widely applied to reconstruct the sea surface temperature at which alkenones were synthesized (Müller et al., 1998). The molecular phylogenetic analysis of environmental samples revealed that various Isochrysidaceae strains are distributed in saline lakes and brackish waters (Theroux et al., 2010; Toney et al., 2012).

Long chain alkenones (LCAs) are well preserved in bottom sediments. The alkenone distribution in lakes differs from the alkenone distribution in the marine realm. For example, the alkenone C37:3 predominates the alkenone distribution in marine environments whereas C37:4 is a more abundant alkenone in lake environments. The salinity of the aquatic environment and the species composition of alkenone producers are important factors affecting the ratio of unsaturated hydrocarbons. Moreover, it was found that the content of tetra-unsaturated alkenones decreases with increasing salinity of the aquatic medium. In recent years, LCAs have become widely used for the reconstruction of the paleoclimate of continental lakes.

Therefore, the main purpose of the study is to identify the quantitative and qualitative composition of long-chain alkenones in the upper layers of the bottom sediments of ten salt lakes in Southern Siberia and to assess the possibility of using this class of compounds as a salt-sensitive bio-marker for paleoclimatic reconstructions in the corresponding regions.

\section{Materials and methods}

The bottom sediments cores of 10 lakes were collected at the end of July 2019 using a corer sampler UWITEC (Austria) with a plastic pipe $(90 \mathrm{~mm})$. Then, 1 $\mathrm{cm}$ thick top layer was separated from each core. The samples were placed in separate sealed plastic bags and stored at a temperature of $-20{ }^{\circ} \mathrm{C}$. Alkenones were extracted in a solution of chloroform and methanol (7: 3 by volume) at room temperature for 24 hours according to the method of extraction of alkenones (Randlett et al.,2014), adapted to our facility and laboratory equipment. Unsaponifiable compounds were separated on a GC-MS chromatograph (Agilent Technologies, 7890/5975C, USA) using an HP-5MS capillary column and Sterin-HP method (M/AMSL, internal standard hexatriacontane C36).

*Corresponding author.

E-mail address: bulkhinlive@yandex.ru (A.O. Bulkhin)

(C) Author(s) 2020. This work is distributed under the Creative Commons Attribution 4.0 License. 


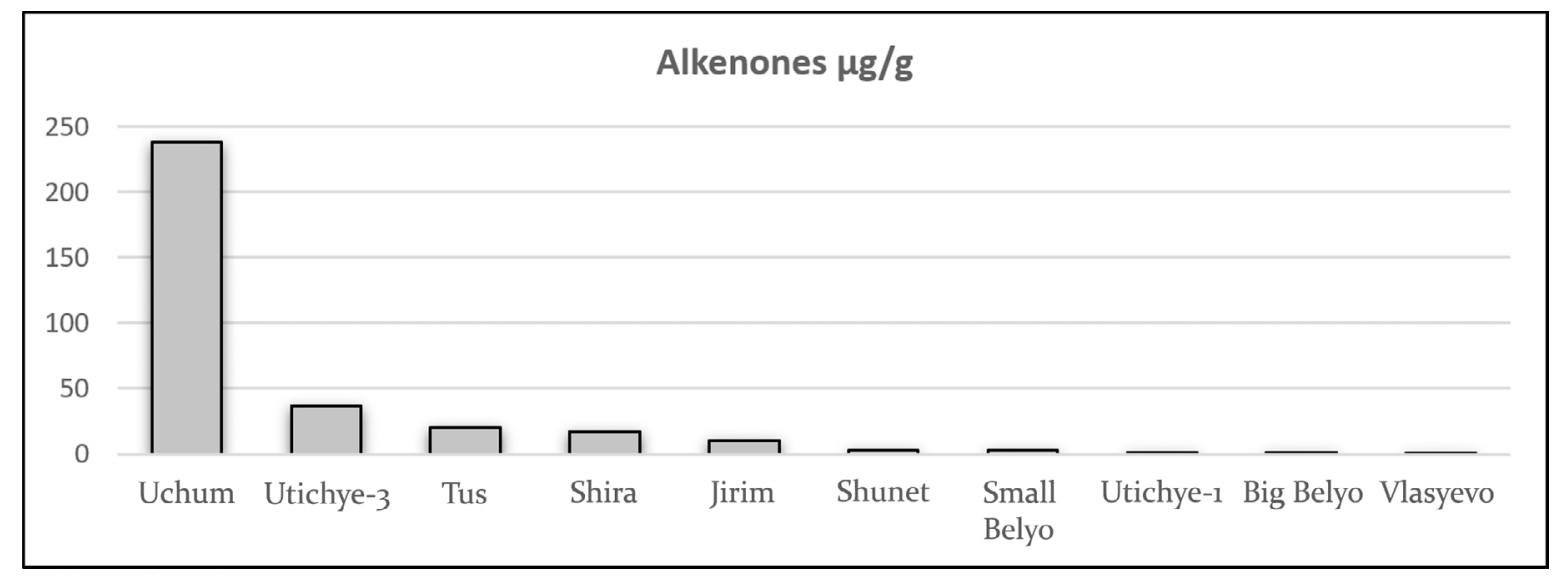

Fig.1. The total content of LCAs in the upper layers of the bottom sediments of lakes

\section{Results and discussion}

All 10 samples of the upper layers of the bottom sediments contained detectable concentrations of LCAs. The distribution of total LCAs content among the lakes was significantly different (Fig. 1).

With an almost equal indicator of organic content, the maximum concentration of alkenones was observed in Lake Uchum $237.7 \mu \mathrm{g} / \mathrm{g}$ and the minimum in Lake Vlasyevo $0.2 \mu \mathrm{g} / \mathrm{g}$. The chain length of alkenones ranged from 37 to 40 carbon atoms. Fractions with 37, 38 and 39 carbon atoms are represented by compounds with 2 , 3 and 4 trans double bonds, and C40 with only 2 and 3 trans double bonds. Alkenones with 37 carbon atoms dominate in all fractions of the samples. Alkenones C40 were found only in two places in Lakes Uchum and Tus with the smallest share in the LCAs fractions (Fig. 2), which is explained by increased salinity of $22 \mathrm{~g} / 1$ and $44 \mathrm{~g} / 1$, respectively.

Alkenone C37: 4 is present in considerable concentrations in all the upper layers of the bottom sediments of the studied lakes, and occupies a large part among the fractions except Lake Shunet. The correlation coefficient $\mathrm{R}^{2}$ of the connection of alkenone C37:4 with salinity is positive and equal to 0.7 (Fig. 3). This fact means that the synthesis and relative distribution of C37: 4 alkenone in alkenone producers depends on the environment and can serve as a marker of salinity in paleo-climatic reconstructions.

\section{Conclusions}

Present study revealed the presence of long-chain alkenones in the upper layers of bottom sediments saline lakes and, therefore, confirmed the presence of haptophytic microalgae in lakes of Southern Siberia region. Polyunsaturated alkenones with 37 and 38 carbon atoms with 2, 3 and 4 double bonds are the main compounds of the extractable fractions of alkenones. Alkenones C40 with 2 and 3 double bounds were found only in two lakes with high salinity compared to other lakes. The correlation coefficient of the connection of alkenone C37:4 with salinity means that C37: 4 can serve as a bio-marker of salinity in paleo-climatic reconstructions.

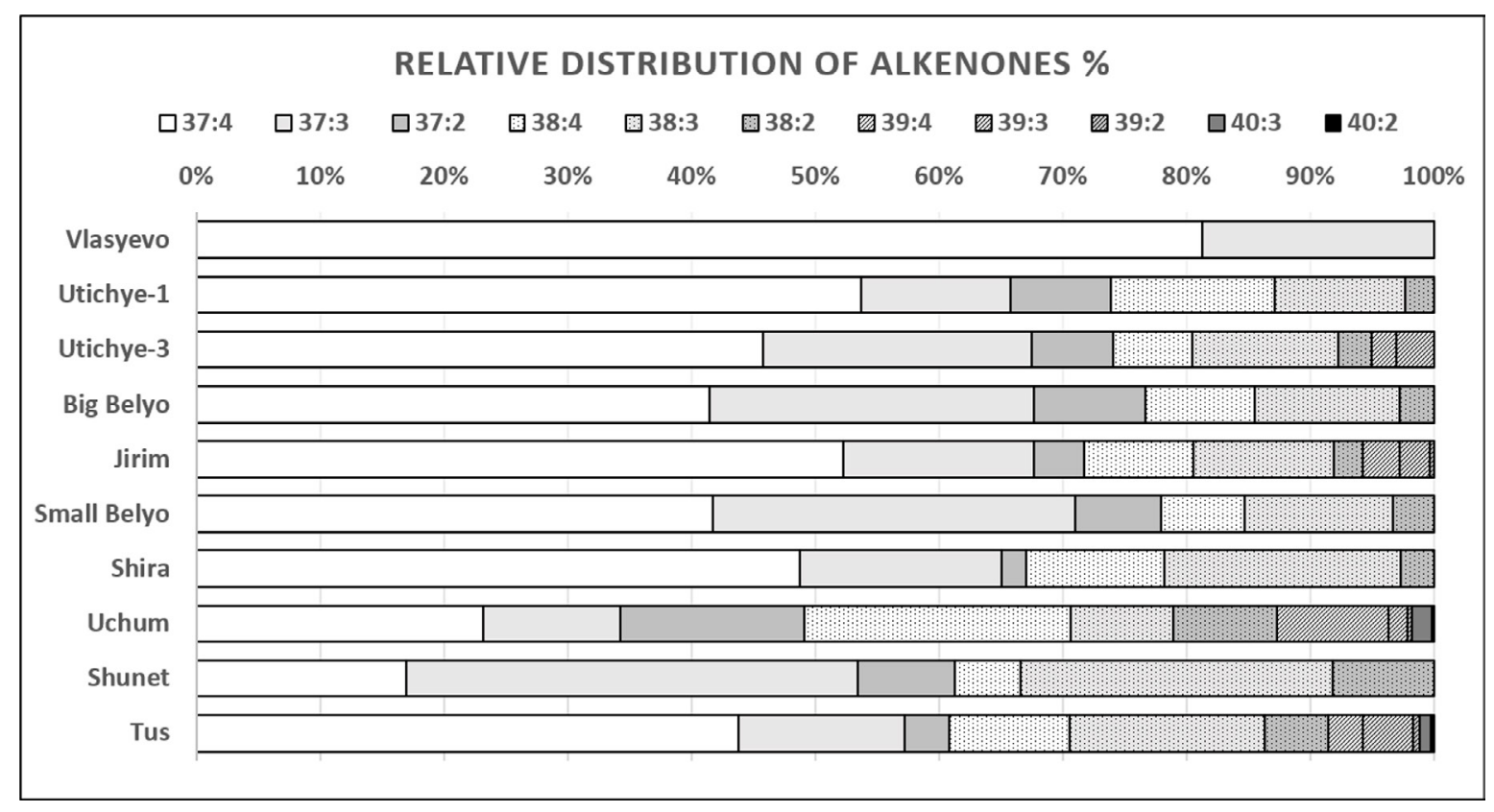

Fig. 2 Relative distribution of LCAs in the upper layers of lake bottom sediments 


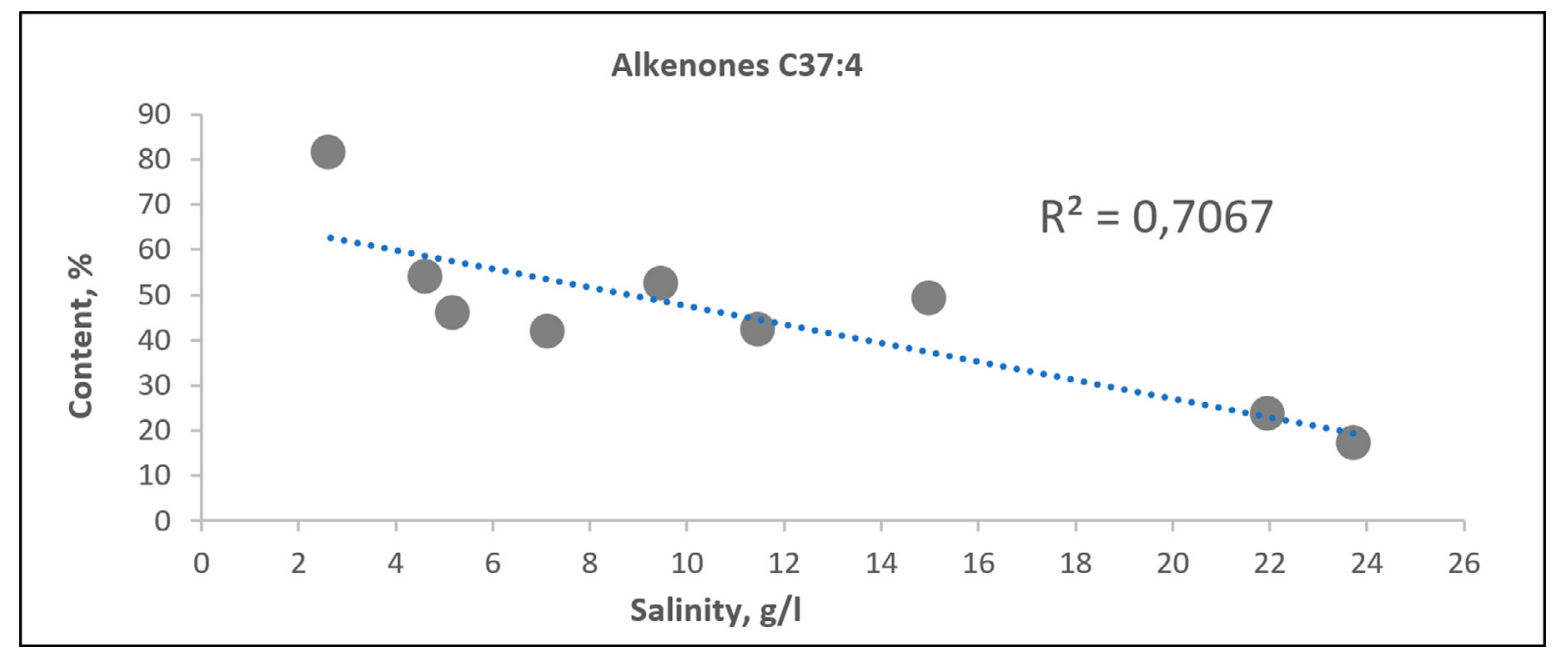

Fig. 3 Correlation analysis of alkenone C37:4 in the upper layers of bottom sediments with salinity among lakes

\section{Acknowledgements}

The reported study was funded by Russian Foundation for Basic Research № 19-05-00428.

\section{References}

Araie H., Nakamura H., Plancq J., Shiratori T., Seki O., Ishida K., Suzuki I. 2018. Novel alkenone-producing strains of genus Isochrysis (Haptophyta) isolated from Canadian saline lakes show temperature sensitivity of alkenones and alkenoates. Organic Geochemistry 121: 89-103. DOI:10.1016/j.orggeochem.2018.04.008

Müller, P.J., Kirst, G., Ruhland, G., Storch von, I., Rosell-Mele, A. 1998. Calibration of the alkenone paleotemperature index UK'37 based on core-tops from the eastern South Atlantic and the global ocean $(60 \mathrm{oN}-60 \mathrm{oS})$.
Geochimica et Cosmochimica 62: 1757-1772. DOI: 10.1016/ S0016-7037(98)00097-0

Randlett M., Stockhecke M., Pickarski N., Balkema C. 2014. Alkenone distribution in Lake Van sediment over the last $270 \mathrm{ka}$ influence of temperature and haptophyte species composition. Quaternary Science Reviews 104: 53-62. DOI:10.1016/j.quascirev.2014.07.009

Theroux, S., D'Andrea, W.J., Toney, J., Amaral-Zettler, L., Huang, Y. 2010. Phylogenetic diversity and evolutionary relatedness of alkenone-producing haptophyte algae in lakes: implications for continental paleotemperature reconstructions. Earth and Planetary Science Letters 300: 311-320. DOI: 10.1016/j.epsl.2010.10.009

Toney, J.L., Huang, Y., Fritz, S.C., Baker, P.A., Grimm, E., Nyren, P. 2010. Climatic and environmental controls on the occurrence and distributions of long chain alkenones in lakes of the interior United States. Geochimica et Cosmochimica 74: 1563-1578. DOI: 10.1016/j.gca.2009.11.021 